\title{
ARTICLE \\ Compound LM9, a novel MyD88 inhibitor, efficiently mitigates inflammatory responses and fibrosis in obesity-induced cardiomyopathy
}

\author{
Xu-yong Zheng ${ }^{1,2}$, Chu-chu Sun ${ }^{3}$, Qian Liu ${ }^{1,2}$, Xiao-yao Lu ${ }^{1}$, Li-li Fu ${ }^{1}$, Guang Liang ${ }^{1}$, Xiu-hua Zhang ${ }^{2}$ and Gao-zhi Chen ${ }^{1}$
}

\begin{abstract}
Mechanisms of cardiomyopathy caused by obesity/hyperlipidemia are complicated. Obesity is usually associated with chronic low-grade inflammation and may lead to the onset and progression of myocardial fibrosis and remodeling. TLR4/ MyD88 signaling pathway, as a key regulator of inflammation, plays an important role in the pathogenesis of obesity-induced cardiomyopathy. We previously demonstrated that LM9, a novel MyD88 inhibitor, attenuated inflammatory responses and fibrosis in obesity-induced cardiomyopathy by inhibiting the formation of TLR4/MyD88 complex. In this study, we investigated the protective effects of LM9 on obesity-induced cardiomyopathy in vitro and in vivo. We showed that LM9 $(5,10 \mu M)$ significantly attenuates palmitic acid (PA)-induced inflammation in mouse peritoneal macrophages, evidenced by decreased expression of proinflammatory genes including TNF- $a$, IL-6, IL-1 $\beta$, and ICAM-1. In cardiac-derived H9C2 cells, LM9 treatment suppressed PA-induced inflammation, lipid accumulation, and fibrotic responses. In addition, LM9 treatment also inhibited PA-activated TLR4/MyD88/NF-KB signaling pathway. We further revealed in HEK293 cells that LM9 treatment blocked the TLR4/ MyD88 binding and MyD88 homodimer formation. In HFD-fed mice, administration of LM9 (5, $10 \mathrm{mg} / \mathrm{kg}$, ig, every other days for 8 weeks) dose-dependently alleviated inflammation and fibrosis in heart tissues and decreased serum lipid concentration. In conclusion, this study demonstrates that MyD88 inhibitor LM9 exerts protective effects against obesityinduced cardiomyopathy, suggesting LM9 to be a promising therapeutic candidate drug for the obesity-related cardiac complications.
\end{abstract}

Keywords: MyD88; inflammation; obesity; cardiomyopathy; palmitic acid

Acta Pharmacologica Sinica (2020) 41:1093-1101; https://doi.org/10.1038/s41401-020-0410-x

\section{INTRODUCTION}

Obesity incidence has increased each year, and it has become a global public health problem [1]. Obesity leads to an inflammatory condition, causing elevated levels of inflammatory cytokines and disorders of immune function, and is directly involved in the etiology of cardiovascular diseases, type 2 diabetes mellitus, and certain types of cancer $[2,3]$. Moreover, a large number of studies have demonstrated that obesity is one of the independent risk factors for myocardial dysfunction and remodeling, leading to an increase in the incidence and mortality of cardiovascular disease [4-6]. Several mechanisms have been identified in obesity-induced cardiomyopathy, including oxidative stress, autophagy, and adrenergic and renin-angiotensin aldosterone overflow [7-9]. Chronic lowgrade inflammation has also been indicated as an important process in obesity-induced cardiomyopathy.

There is increasing interest in the link between obesity and inflammation $[10,11]$. Due to the obesity process, ongoing chronic inflammation causes infiltration of inflammatory cells and progressive changes in tissue destruction and remodeling
$[3,12]$. Moreover, the link between obesity and fibrosis of the myocardium has been well determined $[13,14]$. The levels of free fatty acids (FFAs), especially saturated fatty acids (SFAs), are significantly elevated in obese patients [15] and cause an inflammatory response, which is an important factor in the development of obesity-related cardiovascular diseases [16, 17]. In particular, our previous study reported that palmitic acid (PA), the most abundant circulating SFA, induces myocardial inflammatory injury through the Toll-like receptor 4 (TLR4) accessory protein MD2 in vitro and in vivo [18].

TLR4 is an essential modulator of innate immunity and links innate immunity and metabolic disorders, such as obesity. Myeloid differentiation protein 88 (MyD88) is one of the major adapterdependent downstream signaling pathways of activated TLR4. Once bound to LPS or PA, the MD2 protein induces the dimerization of TLR4 and then recruits MyD88 and produces many proinflammatory molecules. Increasing evidence indicates that the TLR4/MyD88 signaling pathway not only plays an important role in the inflammatory response to infection but also participates in the development of metabolic-related diseases

\footnotetext{
${ }^{1}$ Chemical Biology Research Center, School of Pharmaceutical Sciences, Wenzhou Medical University, Wenzhou 325035, China; ${ }^{2}$ Department of Pharmacy, The First Affiliated Hospital of Wenzhou Medical University, Wenzhou 325035, China and ${ }^{3}$ The Third Affiliated Hospital of Wenzhou Medical University, Ruian 325200, China Correspondence: Xiu-hua Zhang (wzzhangxiuhua@126.com) or Gao-zhi Chen (cgztc@163.com)

These authors contributed equally: Xu-yong Zheng, Chu-chu Sun
}

Received: 17 November 2019 Accepted: 27 March 2020

Published online: 27 April 2020 
1094

such as cardiovascular diseases $[19,20]$. Targeting MyD88 may be an effective strategy for the treatment of obesity-induced cardiomyopathy.

Thus, in the current study, we provide evidence that compound LM9, a novel MyD88 inhibitor from our previous study [21, 22], efficiently attenuates inflammatory responses and fibrosis in obesity-induced cardiomyopathy by inhibiting the formation of the TLR4/MyD88 complex. We suggest that LM9 may be a promising therapeutic candidate drug for obesity-related cardiac complications.

\section{MATERIALS AND METHODS}

Chemicals and reagents

LM9 was synthesized with a purity over $97 \%$ in our laboratory. PA was solubilized in low-endotoxin bovine serum albumin (BSA) $(5 \%)$ at a final concentration of $5 \mathrm{mM}$. PA and BSA were purchased from Sigma-Aldrich (St. Louis, MO, USA). The MTT assay kit and oil red $O$ staining kit (for cultured cells) were acquired from Solarbio (Beijing, China). Antibodies were purchased from the following suppliers: anti-lamin B1 (ab133741), anti-IkB alpha (ab133462), anti-Ly6G (ab25377), anti-TNF-a (ab6671), anti-collagen I (ab6308), and anti-collagen IV (ab6586) were obtained from Abcam (Cambridge, MA, USA). Anti-TLR4 (sc-293072) and anti-TGF- $\beta$ (sc130348) were obtained from Santa Cruz (Dallas, TX, USA). AntiFLAG (SAB4200071) and anti-HA (MFCD00803873) were purchased from Sigma-Aldrich (St. Louis, MO, USA). Anti-ICAM-1 (Cat\# 602991-lg) was obtained from Proteintech (Wuhan, China). Two plasmids encoding pCMV-HA-MyD88 and Flag-MyD88 were obtained from Sino Biological Inc. (Beijing, China).

ELISA kits for mouse TNF- $\alpha$, IL- $1 \beta$, and IL- 6 were purchased from Invitrogen (Carlsbad, CA, USA). Nuclear and cytoplasmic protein extraction kits were from KeyGEN Biotech (Nanjing, China). PrimeScript ${ }^{\mathrm{TM}}$ RT reagent Kit with gDNA Eraser (Perfect Real Time) was purchased from Takara Bio (RR047A, Shiga, Japan).

\section{Compound solution preparation}

LM9 was dissolved in DMSO to a final drug concentration of $20 \mathrm{mM}$ and stored at $-20^{\circ} \mathrm{C}$. Before cell experiments, the LM9 stock solution was diluted to $5 \mathrm{mM}$ and $10 \mathrm{mM}$ working solutions with DMSO. The final concentration of DMSO in the cell experiments did not exceed $0.1 \%(v / v)$. In animal experiments, $1 \%$ sodium carboxymethylcellulose solution was used to formulate LM9 into a 0.5 or $1 \mathrm{mg} / \mathrm{L}$ suspension. The control group and the model group were given equal doses of $1 \%$ sodium carboxymethylcellulose solution.

Primary macrophage isolation and cell culture

C57BL/6J mice were injected i.p. with $3 \mathrm{~mL}$ of $6 \%$ thioglycollate solution $(0.3 \mathrm{~g}$ beef extract, $1.0 \mathrm{~g}$ tryptone, and $0.5 \mathrm{~g} \mathrm{NaCl}$ dissolved in $100 \mathrm{~mL}$ double-distilled $\mathrm{H}_{2} \mathrm{O}$ and filtered through a $0.22-\mu \mathrm{m}$ filter). After $48 \mathrm{~h}$, the mice were sacrificed, and peritoneal cavities underwent lavage with $10 \mathrm{~mL}$ RPMI-1640 medium. Samples were centrifuged and resuspended in RPMI-1640 medium with $10 \% \mathrm{FBS}, 100 \mathrm{U} / \mathrm{mL}$ penicillin $\mathrm{G}$ and $100 \mathrm{mg} / \mathrm{mL}$ streptomycin. Non-adherent cells were removed $2 \mathrm{~h}$ after seeding.

H9C2 myoblasts and HEK293T cells were purchased from the Cell Bank of the Chinese Academy of Sciences (Shanghai, China) and American Type Culture Collection (ATCC, Manassas, VA, USA), respectively. The cells were cultured in DMEM supplemented with $10 \%(v / v)$ FBS, $100 \mathrm{U} / \mathrm{mL}$ penicillin $\mathrm{G}$, and $100 \mathrm{mg} / \mathrm{mL}$ streptomycin. The cells were maintained at $37^{\circ} \mathrm{C}$ under a humidified atmosphere of $5 \% \mathrm{CO}_{2}$ in an incubator.

Animals

Male C57BL/6J mice weighing 18-22 $\mathrm{g}$ were purchased from the Wenzhou Medical University Animal Center (Wenzhou, China). The mice were housed in a standard, pathogen-free animal facility under a $12 \mathrm{~h}$ light/dark cycle at $22-24^{\circ} \mathrm{C}$ with unrestricted access to food and water for the duration of the experiment. All animal experimental protocols were reviewed and approved by the Wenzhou Medical University Animal Policy and Welfare Committee. Obesity was induced by feeding the mice an HFD (D12492) composed of $60 \%$ fat, $20 \%$ protein, and $20 \%$ carbohydrate (Catalogue No. MD12033; MediScience Diets, Yangzhou, China). Control mice were fed a standard control diet containing $10 \%$ fat, $20 \%$ protein, and $70 \%$ carbohydrate (Catalogue No. D12450B, Research Diets, Yangzhou, China). The mice in this experiment were randomly divided into four groups as follows: (1) the control group $(n=6)$; (2) the HFD group $(n=7)$; (3) the $5 \mathrm{mg} / \mathrm{kg}$ LM9treated HFD group $(n=7)$; and (4) the $10 \mathrm{mg} / \mathrm{kg}$ LM9-treated HFD group $(n=7)$. The mice were fed a high-fat diet or a standard diet for 16 weeks, and then the LM9 groups were treated with LM9 every 2 days at a dose of 5 or $10 \mathrm{mg} / \mathrm{kg}$ for 8 weeks by intragastric injection. Weekly monitoring of body weight beginning at the fourth week was performed. At the end of the study, the mice were killed by cervical dislocation after anesthetization with $4 \%$ chloral hydrate. Then, blood and heart tissues were collected.

Cell viability

Cell viability was measured by using the MTT assay. Cells were cultured in 96 -well plates at a density of $6 \times 10^{3}$ cells per well overnight and were subsequently treated with different concentrations of the indicated compound for $24 \mathrm{~h}$. Then, $20 \mu \mathrm{L} \mathrm{MTT}$ solution $(5 \mathrm{mg} / \mathrm{mL})$ was added to each well. After $4 \mathrm{~h}$, the medium was discarded, $150 \mu \mathrm{L}$ DMSO was added, and the absorbance was measured at $490 \mathrm{~nm}$. Cell viability was defined as the percentage of the absorbance compared with the control.

\section{ELISA}

After pretreatment with LM9 (5 and $10 \mu \mathrm{M})$ for $1 \mathrm{~h}$, MPMs were then incubated with PA $(200 \mu \mathrm{M})$ for $24 \mathrm{~h}$. The samples were subsequently centrifuged at $12,000 \mathrm{rpm}$ for $10 \mathrm{~min}$ at $4{ }^{\circ} \mathrm{C}$. Supernatants from cell cultures were assayed for mouse tumor necrosis factor alpha (TNF-a) and interleukin-6 (IL-6) according to the manufacturer's instructions (Invitrogen). The levels of the proteins were calculated using the standard curve method.

Analysis of serological markers

Colorimetric assays were performed using a colorimetric assay kit for non-esterified fatty acids and creatine kinase, including triglycerides (TGs, A110-1-1), total cholesterol (TCH, F002-1-1), high-density lipoprotein (HDL, A112-1-1), and creatine kinase (CK, A032-1-1). Creatine kinase MB isoenzyme (CK-MB, H197) was determined using a CK-MB ELISA kit. All of the kits were purchased from Nanjing Jiancheng BioTech (Nanjing, China).

\section{Histology and immunohistochemistry}

Heart tissues were fixed in $4 \%$ formalin for $24 \mathrm{~h}$ at RT. After embedding in paraffin, the heart samples were cut into $5 \mu \mathrm{m}$ sections, which were prepared and stained with H\&E or Sirius red using standard procedures.

The sections for immunohistochemistry were deparaffinized, rehydrated and then incubated with $3 \%$ hydrogen peroxide for 30 min and blocked with $1 \%$ BSA for $30 \mathrm{~min}$ at room temperature. The sections were then incubated with anti-Ly6G or anti-TNF-a antibodies at $4{ }^{\circ} \mathrm{C}$ overnight. Next, the sections were incubated with horseradish peroxidase-conjugated secondary antibodies. Histochemical visualization was carried out with DAB. Images were captured using a Nikon microscope equipped with a digital camera (Tokyo, Japan).

\section{Coimmunoprecipitation assay}

Cells were seeded in $60 \mathrm{~mm}$ cell culture dishes at a density of $1 \times 10^{6}-2 \times 10^{6}$ cells per well and cultured overnight for coimmunoprecipitation assays. The cells were collected and lysed in lysis 
buffer (AR0101/0103, Boster Biological Technology Co. Ltd, Pleasanton, CA, USA) and centrifuged at $12,000 \mathrm{rpm}$ for $10 \mathrm{~min}$ at $4{ }^{\circ} \mathrm{C}$. Two microliters of antibody were added to $600 \mu \mathrm{g}$ of protein, and the samples were gently rotated at $4{ }^{\circ} \mathrm{C}$ overnight. The immune complexes were collected with protein $\mathrm{A}+\mathrm{G}$ agarose beads (P2012, Beyotime Institute of Biotechnology, Haimen, China), and the precipitates were washed five times with ice-cold PBS. Prewashed agarose beads were boiled in sample buffer for $10 \mathrm{~min}$.

Western blot analysis

Proteins were extracted from the heart tissue or cells using lysis buffer (AR0101/0103, Boster Biological Technology Co. Ltd, Pleasanton, CA, USA) according to the manufacturer's instructions. The proteins were separated by SDS-polyacrylamide gel electrophoresis, transferred to polyvinylidene difluoride membranes (PVDF, Bio-Rad, Hercules, CA, USA), blocked with $8 \%$ skim milk (BD, Franklin Lakes, NJ, USA) and exposed to primary antibody overnight $\left(4^{\circ} \mathrm{C}\right)$, followed by horseradish peroxidase-labeled secondary antibodies for another $1 \mathrm{~h}$. The immune complexes were visualized by exposure in a ChemiDoc XRS + system (BioRad, Hercules, CA, USA).

Quantitative real-time PCR

Total RNA from heart tissues or cells was extracted using TRIzol reagent (Takara Bio, Shiga, Japan). Reverse transcription was performed using the PrimeScript ${ }^{\mathrm{TM}}$ RT reagent kit. qRT-PCR was performed using SYBR Green Supermix (Bio-Rad, Hercules, CA, USA). Primers including TNF- $\alpha$, IL-6, IL-1 $\beta$, ICAM-1, BNP, TGF- $\beta 1$, collagen I, collagen IV, and $\beta$-actin were purchased from Invitrogen. The primer sequences used are shown in Supplementary Table S1.

\section{Statistical analysis}

All data were obtained from at least three independent replicates and calculated as the means \pm standard deviation (mean $\pm S D$ ). The significance of the intergroup differences was analyzed with Student's $t$ tests or one-way analysis of variance
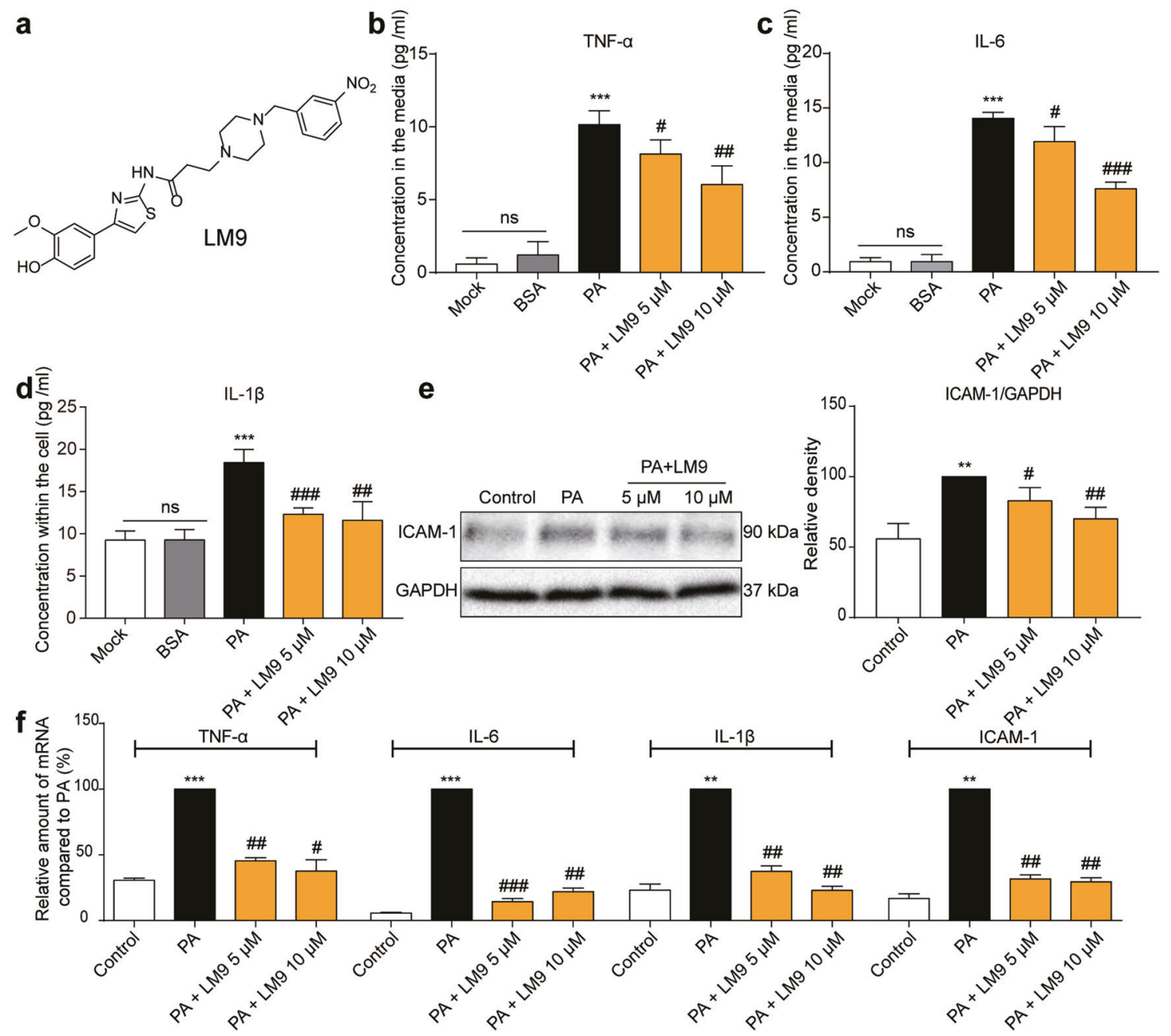
(ANOVA). A $P$ value of $<0.05$ was considered statistically significant.

\section{RESULTS}

LM9 attenuates PA-induced inflammation in mouse peritoneal macrophages

To provide a potential treatment for MyD88-driven inflammation, a new MyD88 inhibitor was synthesized in our laboratory, and the chemical structure of LM9 is shown in Fig. 1a. To determine whether LM9 attenuates PA-induced inflammation, we examined the secretion of TNF- $\alpha$ and IL- 6 and the expression of IL-1 $\beta$ in PAinduced mouse peritoneal macrophages by ELISA (Fig. 1b-d). Our results showed that LM9 reduced PA-induced TNF-a, IL-6, and IL$1 \beta$ in MPMs. In addition, we tested the expression of ICAM-1, and the results indicated that the expression of ICAM- 1 was inhibited by LM9 (Fig. 1e). Then, we tested the mRNA levels of proinflammatory genes in MPMs after treatment with LM9, and we found that LM9 exhibited a dose-dependent inhibitory effect on the mRNA levels of proinflammatory genes, including TNF-a, IL6 , IL-1 $\beta$, and ICAM-1 (Fig. 1f). These results indicate that LM9 restrains $\mathrm{PA}$-induced inflammation.

LM9 suppresses the inflammatory response and NF-KB signaling pathway activation in PA-stimulated $\mathrm{H} 9 \mathrm{C} 2$ cells

The toxicity of LM9 is shown in Fig. 2a. Here, we examined whether LM9 protects against the inflammatory response in PAinduced $\mathrm{H} 9 \mathrm{C} 2$ cells. The qRT-PCR results showed that the mRNA levels of TNF-a, IL-6, ICAM-1, and BNP were significantly reduced in the LM9-treated group (Fig. 2b-e). As the NF-KB signaling pathway

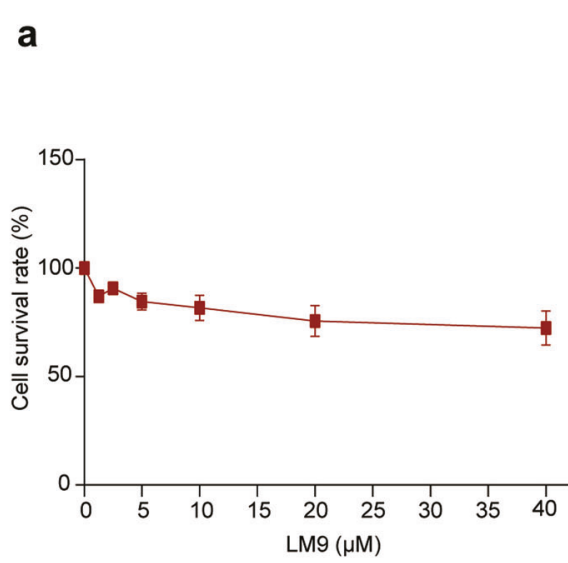

b
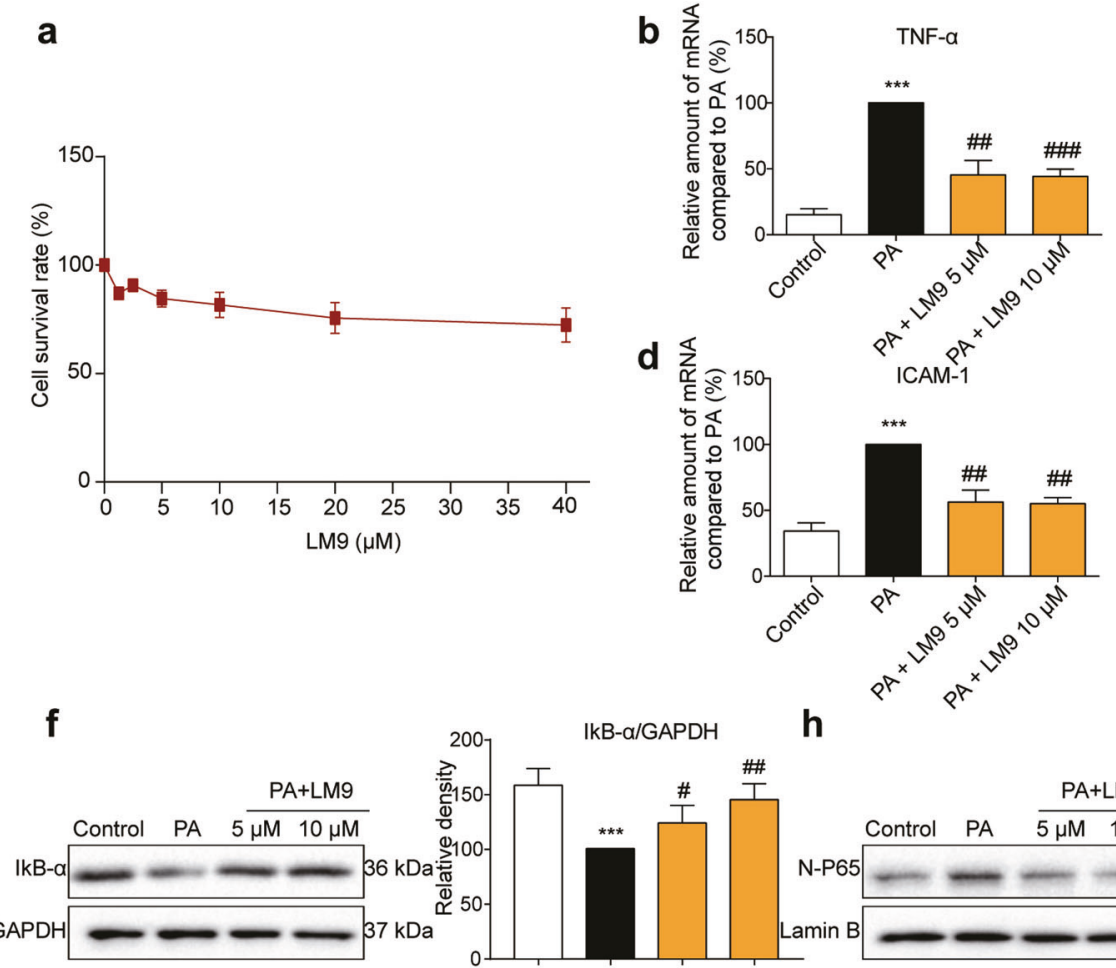

IkB- $\alpha / G A P D H$

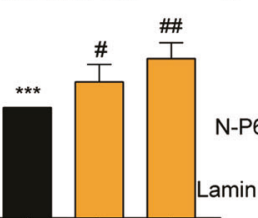

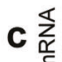
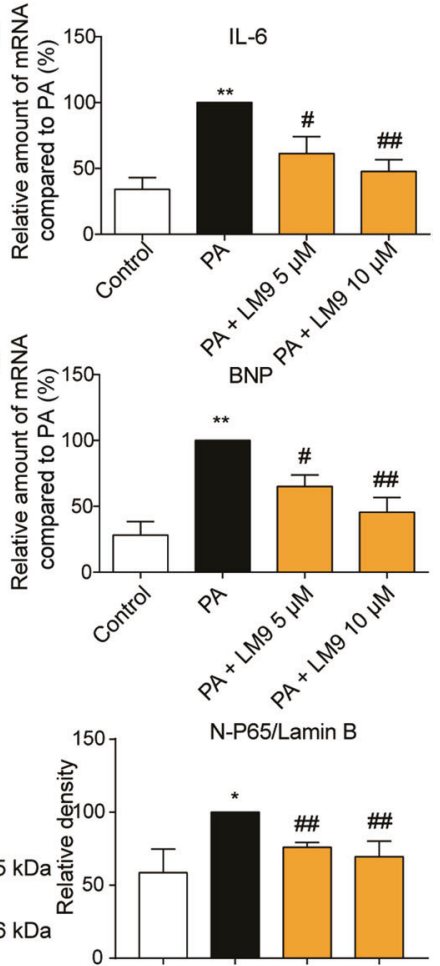

C-P65/GAPDH
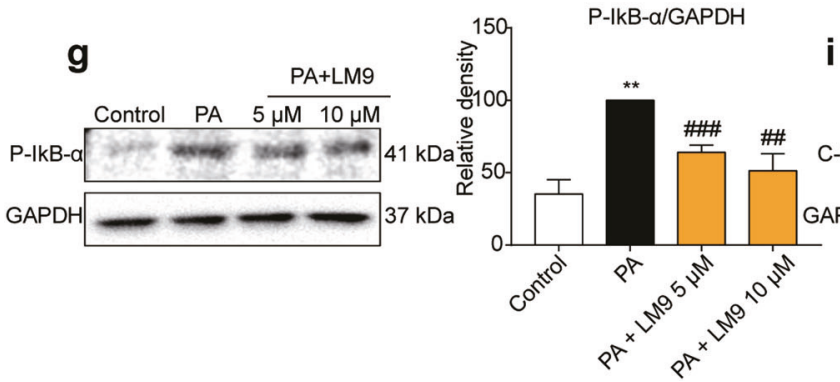

i

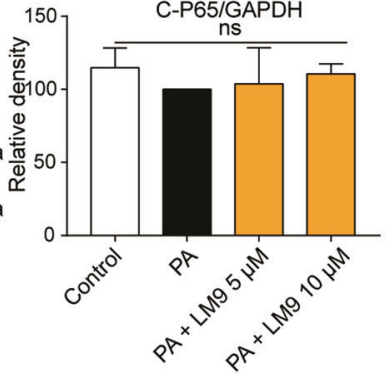

Fig. 2 LM9 suppresses the inflammatory response and NF-KB signaling pathway activation in PA-stimulated H9C2 cells. a The toxicity of LM9 on H9C2 cells based on the MTT assay; the data are expressed as the mean \pm SD $(n=3)$. b-e H9C2 cells were pretreated with LM9 (5 and $10 \mu \mathrm{M}$ ) for $1 \mathrm{~h}$ and stimulated with $200 \mu \mathrm{M}$ PA for $12 \mathrm{~h}$, after which TNF- $\alpha$, IL-6, ICAM-1, and BNP mRNA levels were determined by qRT-

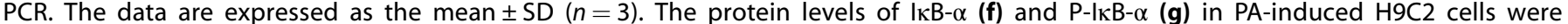
determined by Western blot assay. Representative immunoblots are shown in the upper panel, and densitometric quantification is presented as the mean \pm SD $(n=3)$ in the lower panel. $\mathbf{h}$, $\mathbf{i}$ Western blot of cytosolic and nuclear protein levels of NF- $\mathrm{kB}$ p65 subunit in H9C2 cells that were stimulated with $200 \mu \mathrm{M}$ PA for $1 \mathrm{~h}$. Representative immunoblots are shown in the upper panel, and densitometric quantification is presented as the mean \pm SD $(n=3)$ in the lower panel. Student's $t$ test was utilized for the statistical analysis $(\mathbf{b})-(\mathbf{i})$, and significant differences are indicated as ${ }^{*}$ or ${ }^{\#}$. ${ }^{*} P<0.05$, ${ }^{* *} P<0.01$, and ${ }^{* * *} P<0.001$ vs. the control group; ${ }^{\#} P<0.05$, ${ }^{\# \#} P<0.01$, and ${ }^{\# \# \#} P<$ 0.001 vs. the PA alone group. 


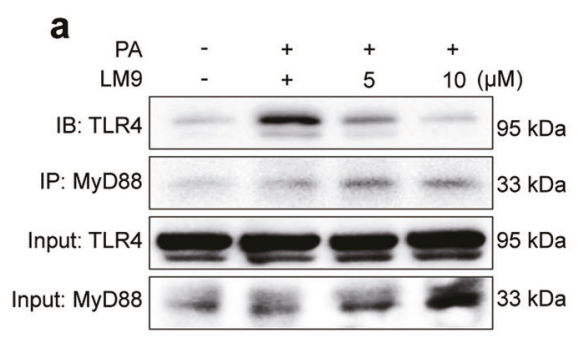

\section{b}

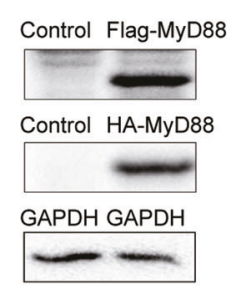

C

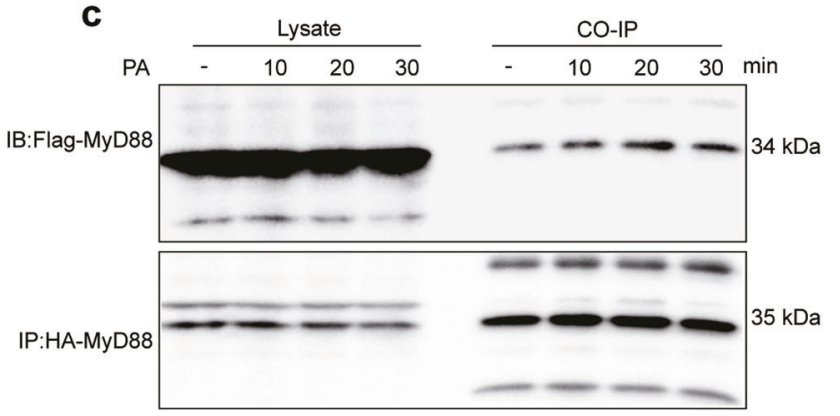



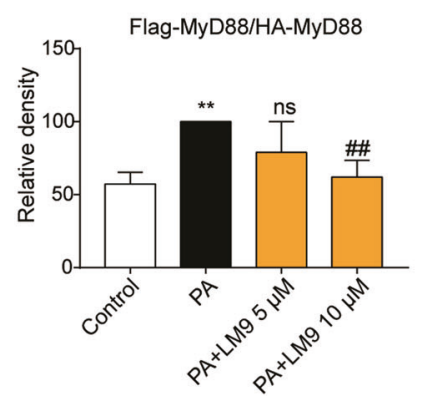

Fig. 3 LM9 inhibits TLR4/NF-KB signaling pathway activation by blocking the TLR4/MyD88 interaction and MyD88 homodimer formation. a Effect of LM9 on the formation of the TLR4/MyD88 complex in HEK293T cells that were stimulated with $200 \mu M$ PA for 50 min. Representative $(n=3)$ Western blots from the coimmunoprecipitation studies are shown. $\mathbf{b}$ The transfection efficacy of HEK293T cells that were cotransfected with HA-MyD88 and Flag-MyD88 for $24 \mathrm{~h}$. Effect of PA-induced MyD88 homodimer formation at 10, 20, and 30 min (c) and the effect of LM9 in blocking MyD88 dimerization (d). The statistical results are shown as the mean \pm SD $(n=3)$ on the right of (d). Student's $t$ test was utilized for the statistical analysis. Significant differences are indicated as ${ }^{*}$ or ${ }^{\#}$. ${ }^{* *} P<0.01$ vs. the control group; ${ }^{\# \#} P<$ 0.01 vs. the PA alone group.

is downstream of TLR4/MyD88 in the inflammatory response, we tested the expression levels of IKB- $\alpha$ and plкB- $\alpha$, and the results indicated that the degradation and phosphorylation of IKB-a were markedly inhibited by LM9 (Fig. 2f, g). In addition, cytosolic and nuclear protein levels of the NF-KB p65 subunit were tested, and we found that LM9 decreased the accumulation of NF-KB p65 in the nucleus (Fig. 2h, i).

LM9 inhibits TLR4/NF-KB signaling pathway activation by blocking TLR4/MyD88 binding and MyD88 homodimer formation We further explored the mechanism by which LM9 inhibits TLR4/ NF-KB activation in PA-induced cells. TLR4/MyD88 binding and MyD88 homodimer formation are necessary for activation of the TLR4/NF-KB signaling pathway $[23,24]$. We found that LM9 treatment significantly inhibited PA-induced TLR4/MyD88 binding based on coimmunoprecipitation assays (Fig. 3a). We next examined whether LM9 inhibited the formation of the MyD88 homodimer. The transfection efficacy of HA-MyD88 and FlagMyD88 plasmids is shown in Fig. 3b. Then, we tested the effect of PA on the formation of the MyD88 homodimer at 10, 20, and 30 min compared with that of the control group, and we found that the MyD88 homodimer apparently increased at 20 min (Fig. 3c). The effects of LM9 on blocking MyD88 dimerization were examined and are shown in Fig. 3d. Thus, LM9 inhibits the TLR4/NF-KB signaling pathway by blocking TLR4/MyD88 binding and MyD88 homodimer formation.

LM9 alleviates PA-induced lipid accumulation and fibrosis in $\mathrm{H} 9 \mathrm{C} 2$ cells

Since cardiac steatosis is associated with impaired cardiac function during obesity, oil red $\mathrm{O}$ staining was utilized to investigate lipid accumulation in $\mathrm{H} 9 \mathrm{C} 2$ cells with different treatments. Our results revealed that LM9 alleviated PA-induced lipid distribution in $\mathrm{H} 9 \mathrm{C} 2$ cells (Fig. 4a, upper panel). Saturated PA induces MD2-dependent inflammatory injury in cardiomyocytes and causes myocardial morphological changes [18]. We measured cellular morphology in $\mathrm{H} 9 \mathrm{C} 2$ cells that were pretreated with LM9 before exposure to PA by hematoxylin staining, and the results indicated that morphological changes in $\mathrm{H} 9 \mathrm{C} 2$ cells were alleviated by LM9 (Fig. 4a, lower panel). Fibrotic change is a feature of obesity-induced cardiomyopathy. We detected the expression levels of profibrotic proteins by Western blotting, and the data showed that the protein levels of collagen $1 / 4$ and TGF- $\beta$ were markedly decreased in $\mathrm{H} 9 \mathrm{C} 2$ cells in the presence of LM9 (Fig. 4b, c). Similar effects were observed when the mRNA levels of profibrotic genes in $\mathrm{H} 9 \mathrm{C} 2$ cells were analyzed by qRT-PCR (Fig. 4d).

Effects of LM9 on body weight, cardiac function, and blood lipid profile in HFD mice

We used an HFD mouse model to investigate whether LM9 exhibits cardioprotective effects in obesity in vivo. HFD feeding gradually and significantly increased the body weights in the HFD group compared with those in the control group. Neither low nor high dose LM9 treatment had effects on HFD feeding-induced body weight increases (Fig. 5a). The cardiac function of mice in four groups was measured. Notably, both treatment groups showed decreased levels of CK-MB and CK compared with those in the HFD group (Fig. 5b, c). We further tested other serological markers that correlate with obesity and lipid metabolism. The levels of serum triglyceride (TG), TCH, HDL, and low-density lipoprotein (LDL) were increased in groups that were fed an HFD but were alleviated in groups that were treated with LM9 (Fig. 5d-g). Moreover, cardiac function was assessed by 


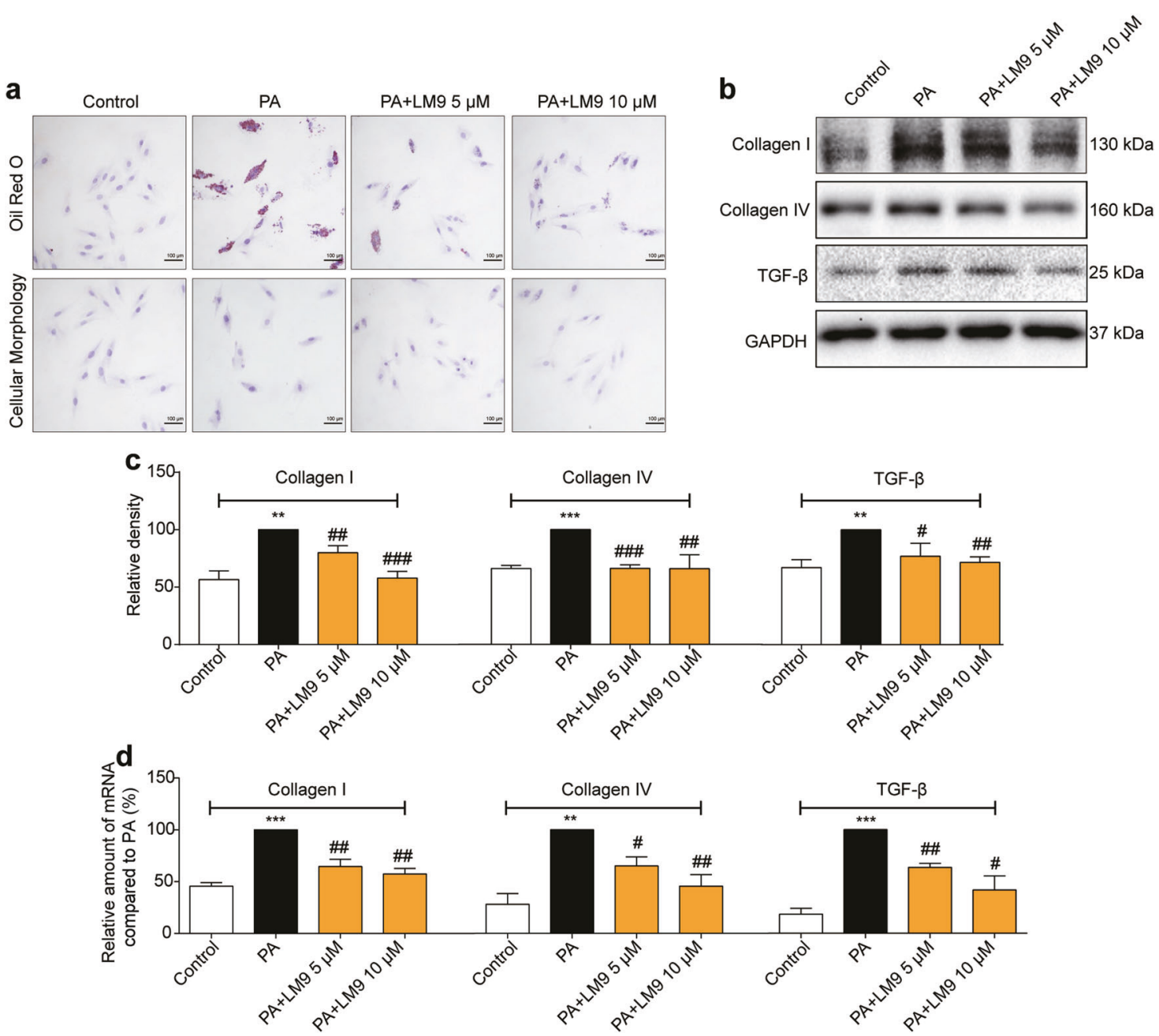

Fig. 4 LM9 alleviates PA-induced lipid accumulation and fibrosis in H9C2 cells. a Representative images of oil red O staining (upper panel) and cellular morphology (lower panel) of H9C2 cells that were stimulated with $200 \mu$ M PA ( $\times 400$ magnification). The protein levels (b) and the densitometric quantification (c) of profibrotic proteins in cells that were pretreated with LM9 before stimulation with $200 \mu \mathrm{M}$ PA for $24 \mathrm{~h}$ were determined by Western blotting (representative of $n=3$, mean \pm SD). d H9C2 cells were pretreated with LM9 (5 and $10 \mu \mathrm{M})$ for $1 \mathrm{~h}$ and stimulated with $200 \mu \mathrm{M}$ PA for $12 \mathrm{~h}$, after which collagen l, collagen IV, and TGF- $\beta$ mRNA levels were determined by qRT-PCR. The data are presented as the mean \pm SD $(n=3)$. Student's $t$ test was utilized for the statistical analysis $(\mathbf{c})$, (d), and significant differences are indicated as * or ${ }^{\#}$. ${ }^{*} P<0.01$ and ${ }^{* * *} P<0.001$ vs. the control group; ${ }^{\#} P<0.05,{ }^{\# \#} P<0.01$, and ${ }^{\# \# \#} P<0.001$ vs. the PA alone group.

echocardiography. Echo examination found that treatment with LM9 improved cardiac function indicators, including EF\% and FS\%, and enhanced left ventricular contractility (Supplementary Table S2).

LM9 mitigates myocardial inflammatory injury and ameliorates fibrosis in HFD mice

To further investigate the effects of LM9, we observed the morphology of the hearts in the four groups by hematoxylin-eosin staining. The results showed that there were significant changes in cardiac structure, as well as myocardial thickening and interstitial proliferation, in the HFD group, while LM9 administration improved the obesity-induced cardiac pathological changes (Fig. 6a, first and second panel). In addition, LM9 ameliorated TNF-a accumulation (Fig. 6a, third panel) and decreased neutrophil infiltration (Fig. 6a, last panel) compared with those in the HFD groups. We also found that LM9 exhibited an obvious inhibitory effect on the mRNA levels of proinflammatory genes, including TNF-a, IL-6, and ICAM-1 (Fig. 6b). Previous studies have suggested that cardiac fibrosis and inflammation are potential contributors to obesity-induced changes in cardiac structure and function [25]. We analyzed the interstitial collagen distribution by picrosirius red staining. As expected, there was significant fibrogenesis in control and HFD mice, while collagen deposition was significantly decreased in both LM9 treatment groups (Fig. 6c). The protein level of IKB-a is shown in Fig. $6 \mathrm{~d}$, and LM9 markedly inhibited the HFD-induced degradation of IKB-a (Fig. $6 \mathrm{~d}$ ). We also examined the expression levels of profibrotic proteins in heart tissue, and our results revealed that LM9 attenuated HFD-induced cardiac fibrosis (Fig. $6 \mathrm{~d}$ and Supplementary Fig. S1). Similar effects were observed when the mRNA levels of profibrotic genes in heart tissue were analyzed (Fig. 6e).

\section{DISCUSSION}

Myocardial inflammation has been widely accepted to play a pivotal role in the physiological and pathological mechanisms of cardiac function and dysfunction [24]. Recent studies have shown that obesity-induced chronic inflammation is associated with the pathophysiology of obesity-related cardiovascular disease [26-28]. Hyperlipidemia leads to increased production of inflammatory cytokines and increased inflammatory cell 

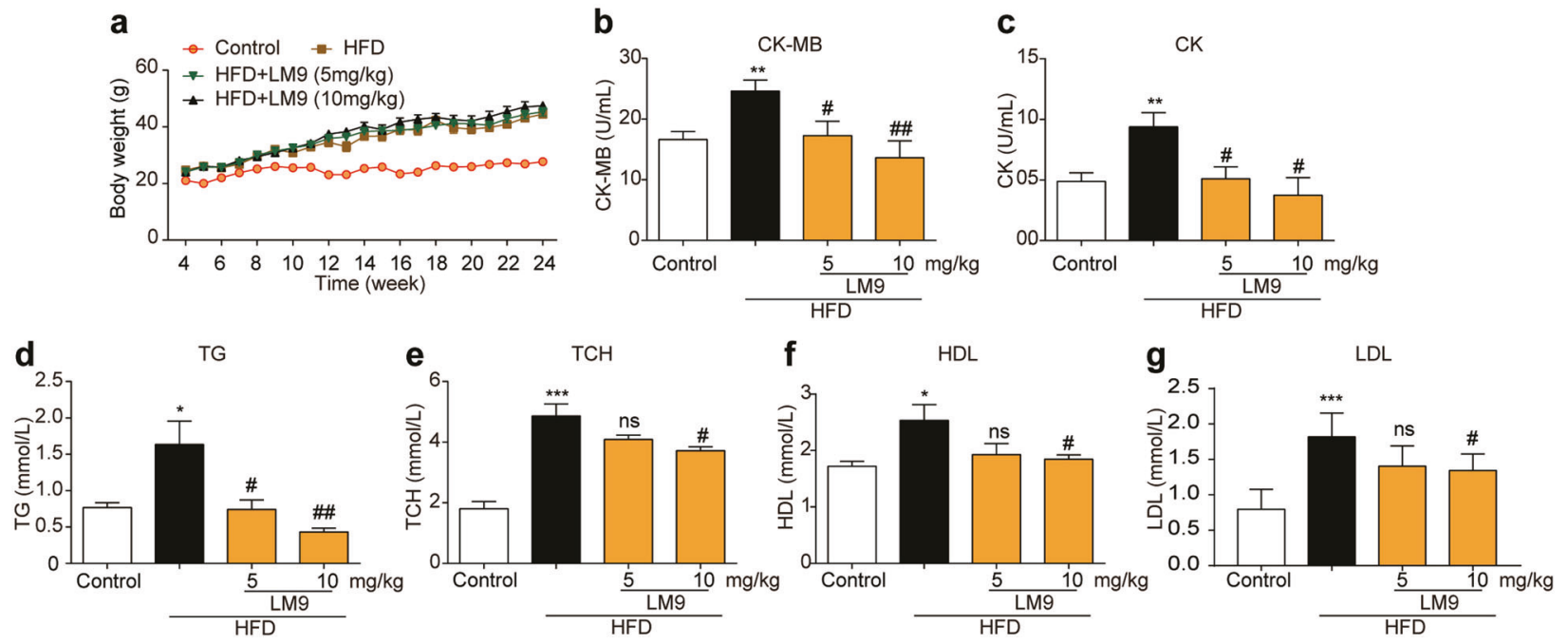

Fig. 5 Effects of LM9 on body weight, cardiac function and blood lipid profile in HFD mice. C57BL/6J mice were fed an HFD for 6 months, and blood was collected for evaluation of myocardial inflammatory injury. a Body weight gain over a 20-week period. b, c Serological markers of myocardium injury were evaluated by CK-MB and CK. $\mathbf{d}-\mathbf{g}$ The blood lipid profile contains triglycerides (TG), total cholesterol (TCH), highdensity lipoprotein (HDL), and low-density lipoprotein (LDL). The data in (a)-(g) are shown as the mean \pm SD, $n=6$ or 7. Student's $t$ test was utilized for the statistical analysis, and significant differences are indicated as ${ }^{*}$ or ${ }^{\#}$. ${ }^{*} P<0.05,{ }^{* *} P<0.01$, and ${ }^{* * *} P<0.005$ vs. the control group; ${ }^{\#} P<0.05,{ }^{\# \#} P<0.01$ vs. the PA alone group.

infiltration, impairing the normal function of cardiomyocytes and leading to myocardial fibrosis and cardiac remodeling [29]. It has been reported that anti-inflammatory drugs alleviate obesityrelated cardiomyopathy in animal models [30-32]. Due to the potential role of inflammation in cardiovascular disease, compounds with anti-inflammatory activity may have unexpected effects in inflammatory-related cardiovascular diseases such as obesity cardiomyopathy.

Notably, numerous studies have demonstrated that TLR4 and MyD88 are robustly expressed in various inflammatory cardiovascular diseases, such as myocarditis, myocardial infarction, ischemia-reperfusion injury, and obesity-associated cardiovascular disease, and TLR4 activates the expression of several proinflammatory cytokine genes through the MyD88-dependent pathway, which plays pivotal roles in myocardial inflammation $[24,33]$. Therefore, we can mitigate obesity-induced cardiomyopathy by targeting TLR4 or its downstream signaling pathways. Currently, the unresolved question is whether it is possible to attenuate deleterious myocardial inflammation and preserve the advantages of innate immunity while also regulating TLR4 signaling [24]. However, directly blocking TLR4 or MD2 is not a perfect strategy for improving myocardial inflammation. By activating the MyD88-independent pathway and further promoting the secretion of type I interferon, which possesses an antiviral effect, the MD2/TLR4 signaling pathway also plays a beneficial role in myocardial inflammation, especially viral myocarditis. Directly inhibiting MD2/TLR4 completely blocks the function of innate immunity. Thus, it is reasonable to find an inhibitor that targets the MyD88-dependent pathway. It has been reported that both heart-specific autoimmunity and MyD88 signaling are critical for cardiac fibrosis and heart failure progression [34]. However, the prevention or treatment of cardiac diseases with MyD88 inhibitors or antagonists to date has not been evaluated in human clinical trials. The lack of favorable MyD88 inhibitors may be a major cause. Recent studies have shown that 2-amino-4-phenylthiazole structures have a variety of pharmacological activities, such as antiinflammatory and antioxidant activities, which can inhibit the dimerization of the MyD88-TIR domain. Our team took this as a lead compound to develop more effective MyD88 inhibitors for the treatment of inflammation, and LM9 is one of the most active compounds with MyD88 binding ability. In LPS-stimulated mouse primary macrophages, LM9 significantly inhibited the TLR4-MyD88 interaction and MyD88 dimerization [21]. Based on the robust anti-inflammatory effects of LM9, our team explored whether LM9 alleviates the low-grade inflammation caused by long-term hyperlipidemia. We found that LM9 inhibited the inflammatory response of mouse primary macrophages induced by SFAs (Fig. 1).

The relationship between obesity and inflammation has been well demonstrated. Obesity is now often associated with chronic low-grade inflammation, suggesting that inflammation may be a potential mechanism leading to obesity-related cardiovascular complications. Moreover, obesity is often accompanied by dyslipoidosis and hyperlipidemia. In obese patients, the level of SFAs is usually elevated, which is directly involved in the etiology of cardiovascular diseases. PA is the main SFA in plasma and stimulates the production of inflammatory cytokines in cultured aortic smooth muscle cells and endothelial cells [18]. Our team found that PA induced the production of inflammatory factors and caused lipid accumulation, morphological changes and fibrosis in $\mathrm{H} 9 \mathrm{C} 2$ cells. However, this pathological state was reversed by LM9 (Figs. 2 and 4). Subsequently, animal experiments further validated the effect of LM9 on myocardial inflammation, morphological changes, lipid accumulation and fibrosis (Figs. 5 and 6).

Lipid accumulation activates harmful signaling pathways, resulting in the production of inflammatory factors and tissue remodeling in cardiomyocytes, leading to obesity-induced cardiomyopathy. The TLR4/MyD88/NF-KB signaling pathway plays a key role in regulating the inflammatory response and is directly related to the release of inflammatory factors and cell adhesion molecules. Our team found that LM9 inhibited PA/ HFD-induced NF-KB signaling pathway activation in cardiomyocytes or animal models, and blocking the NF-KB signaling pathway may be associated with LM9-mediated inhibition of TLR4/MyD88 binding and MyD88 dimerization (Figs. 3 and 6).

In conclusion, our study demonstrates the cardioprotective effects of LM9 on PA- or HFD-induced inflammation and fibrosis 
a
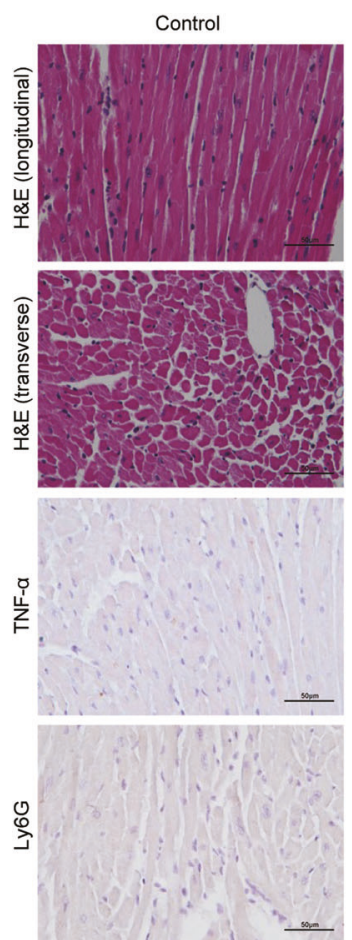

HFD
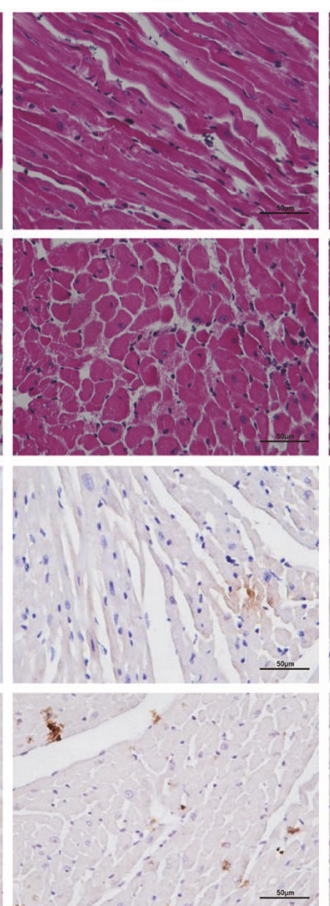

HFD
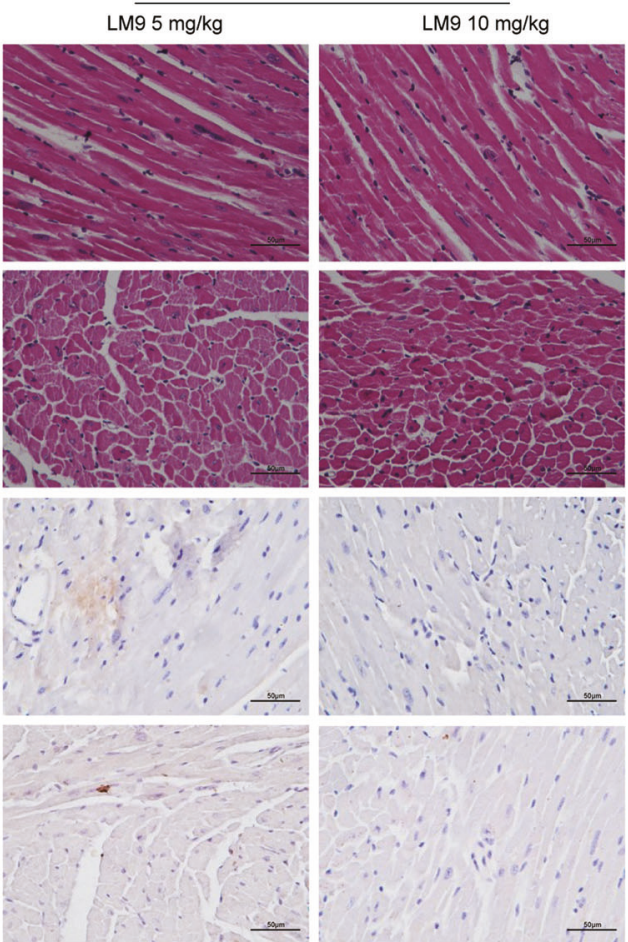

b

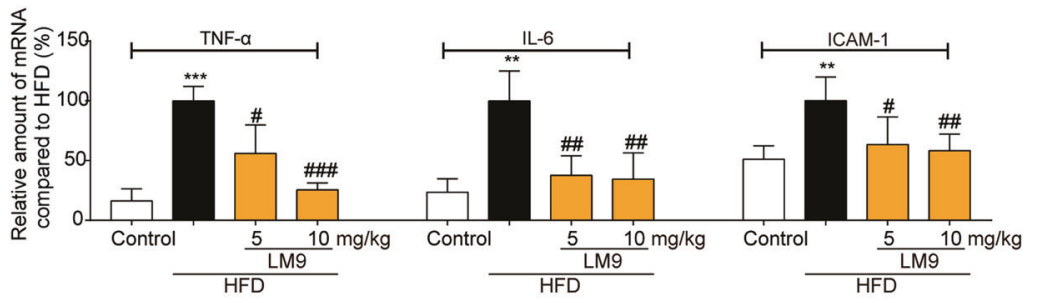

C

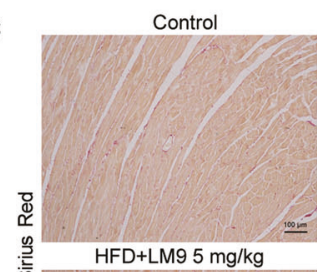

HFD

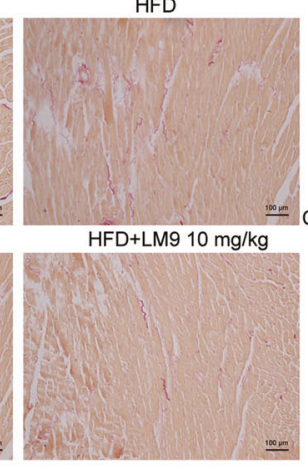

d

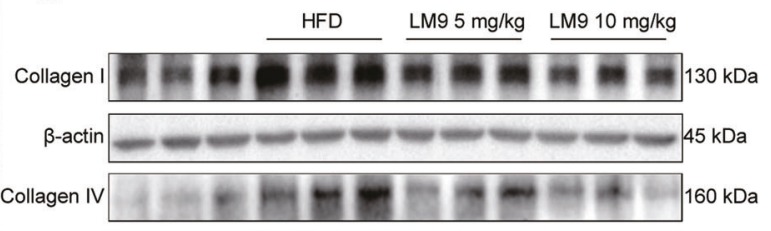

TGF- $\beta=2-\infty-w-w 25 \mathrm{kDa}$

IKB-a

$\beta$-actin

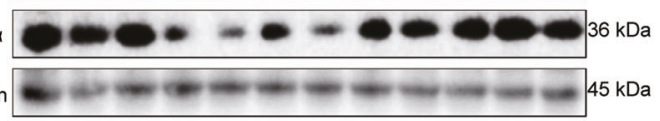

e

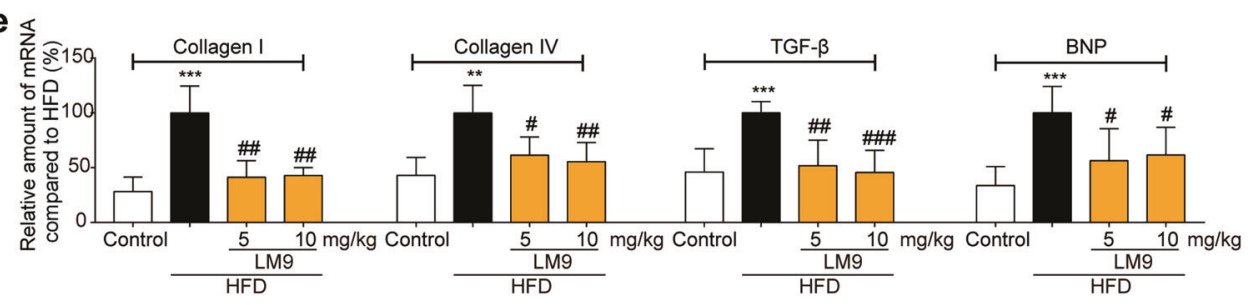

in vitro and in vivo and provides a promising therapeutic drug for the treatment of obesity-related cardiac complications. Inhibition of TLR4/MyD88 may be an effective strategy for the treatment of pathological changes in obesity-induced cardiomyopathy.

\section{ACKNOWLEDGEMENTS}

This work was supported by the National Natural Science Foundation of China (Grant Nos. 81703352 and 81770825) the Clinical Research Project of Zhejiang Medical Association (2017ZYC-A25) and the Zhejiang Medical and Health Science and Technology Plan Project (2019KY448). 
Fig. 6 LM9 mitigates myocardial inflammatory injury and ameliorates fibrosis in HFD mice. C57BL/6J mice were fed an HFD for 6 months, and heart tissue was collected to evaluate myocardial inflammatory injury and amelioration of fibrosis. a The upper panel shows representative myocardium histological changes by hematoxylin and eosin staining; the middle panel shows representative images of IHC analysis of TNF- $\alpha$ accumulation in myocardial tissues; the lower panel shows IHC analysis of Ly6G in myocardial tissues as a marker of inflammatory cell infiltration. Representative $n=3$. b Effects of LM9 on the mRNA levels of proinflammatory genes and adhesion molecules in heart tissue were determined by qRT-PCR. The data are presented as the mean \pm SD. $n \geq 3$. c Representative images of Sirius red staining were used to assess the accumulation of collagen fibers. $\mathbf{d}$ The protein levels of IאB- $\alpha$ and profibrotic proteins in heart tissue were detected by Western blotting (representative of $n=3$ ). e Effects of LM9 on the mRNA levels of profibrotic genes in heart tissue. The data are presented as the mean \pm SD. $n \geq 3$. Student's $t$ test was utilized for the statistical analysis (b), (e), and significant differences are indicated as ${ }^{*}$ or ${ }^{\#} .{ }^{*} P<0.01$,

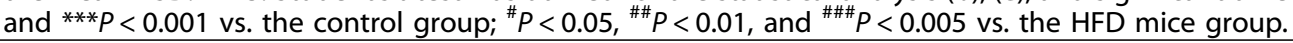

\section{AUTHOR CONTRIBUTIONS}

$\mathrm{XYZ}, \mathrm{XHZ}$, and $\mathrm{GZC}$ designed the research. $\mathrm{XYZ}, \mathrm{CCS}, \mathrm{QL}$, and $\mathrm{XYL}$ performed the research. $X Y Z$ and $G Z C$ wrote the paper. LLF and GL provided the tested compound. $\mathrm{GZC}, \mathrm{XHZ}$, and $\mathrm{GL}$ reviewed and modified the paper.

\section{ADDITIONAL INFORMATION}

The online version of this article (https://doi.org/10.1038/s41401-020-0410-x) contains supplementary material, which is available to authorized users.

Competing interests: The authors declare no competing interests.

\section{REFERENCES}

1. Yanovski JA. Obesity: trends in underweight and obesity - scale of the problem. Nat Rev Endocrinol. 2018;14:5-6.

2. Zarzour A, Kim HW, Weintraub NL. Understanding obesity-related cardiovascular disease: It's all about balance. Circulation. 2018;138:64-6.

3. Rogero MM, Calder PC. Obesity, inflammation, toll-like receptor 4 and fatty acids. Nutrients. 2018;10:432.

4. Gordon-Larsen P, Heymsfield SB. Obesity as a disease, not a behavior. Circulation. 2018;137:1543-5.

5. Kotsis V, Jordan J, Micic D, Finer N, Leitner DR, Toplak H, et al. Obesity and cardiovascular risk: A call for action from the european society of hypertension working group of obesity, diabetes and the high-risk patient and european association for the study of obesity: Part a: Mechanisms of obesity induced hypertension. J Hypertens. 2018;36:1.

6. Fuster JJ, Ouchi N, Gokce N, Walsh K. Obesity-induced changes in adipose tissue microenvironment and their impact on cardiovascular disease. Circ Res. 2016; 118:1786-807.

7. Ali A, Boutjdir M, Aromolaran AS. Cardiolipotoxicity, inflammation, and arrhythmias: role for interleukin-6 molecular mechanisms. Front Physiol. 2018;9:1866.

8. Castaneda D, Gabani M, Choi SK, Nguyen QM, Chen C, Mapara A, et al. Targeting autophagy in obesity-associated heart disease. Obesity. 2019;27:1050-8.

9. Gregor MF, Hotamisligil GS. Inflammatory mechanisms in obesity. Annu Rev Immunol. 2011;29:415-45.

10. Litwin SE. Cardiac remodeling in obesity: time for a new paradigm. JACC Cardiovasc Imaging. 2010;3:275-7.

11. Reilly SM, Saltiel AR. Adapting to obesity with adipose tissue inflammation. Nat Rev Endocrinol. 2017;13:633-43.

12. Isabel BP, Lisa R, J Alfredo M. Oxidative stress and inflammation interactions in human obesity. J Physiol Biochem. 2012;68:701-11.

13. Carroll JF, Tyagi SC. Extracellular matrix remodeling in the heart of the homocysteinemic obese rabbit. Am J Hypertens. 2005;18:692-8.

14. Packer M. Epicardial adipose tissue may mediate deleterious effects of obesity and inflammation on the myocardium. J Am Coll Cardiol. 2018;71: 2360-72.

15. Soriguer F, Garcíaserrano S, Garcíaalmeida JM, Garridosánchez L, Garcíaarnés J, Tinahones FJ, et al. Changes in the serum composition of free-fatty acids during an intravenous glucose tolerance test. Obesity. 2012;17:10-5.

16. Pilz S, Scharnagl H, Tiran B, Seelhorst U, Wellnitz B, Boehm BO, et al. Free fatty acids are independently associated with all-cause and cardiovascular mortality in subjects with coronary artery disease. J Clin Endocrinol Metab. 2006;91:2542.

17. Stefan $P$, Winfried MR. Free fatty acids as a cardiovascular risk factor. Clin Chem Lab Med. 2008;46:429-34.

18. Wang Y, Qian Y, Fang Q, Zhong P, Li W, Wang L, et al. Saturated palmitic acid induces myocardial inflammatory injuries through direct binding to tIr4 accessory protein md2. Nat Commun. 2017:8:13997.

19. Francis K, Matilda P, Ian L, Bannerman DD, Joan T, Joshua T, et al. Toll-like receptor-4 mediates vascular inflammation and insulin resistance in diet-induced obesity. Circ Res. 2007;100:1589-96.

20. Huang S, Rutkowsky JM, Snodgrass RG, Ono-Moore KD, Schneider DA, Newman $J W$, et al. Saturated fatty acids activate tlr-mediated proinflammatory signaling pathways. J Lipid Res. 2012;53:2002.

21. Chen L, Chen H, Chen P, Zhang W, Wu C, Sun C, et al. Development of 2-amino-4 phenylthiazole analogues to disrupt myeloid differentiation factor 88 and prevent inflammatory responses in acute lung injury. Eur J Med Chem. 2019;161:22-38.

22. Chen T, Luo W, Wu G, Wu L, Huang S, Li J, et al. A novel myd88 inhibitor Im9 prevents atherosclerosis by regulating inflammatory responses and oxidative stress in macrophages. Toxicol Appl Pharmacol. 2019;370:44-55.

23. Bovijn C, Desmet AS, Uyttendaele I, Acker TV, Tavernier J, Peelman F. Identification of binding sites for myeloid differentiation primary response gene 88 (myd88) and toll-like receptor 4 in myd88 adapter-like (mal). J Biol Chem. 2013;288:12054-66.

24. Yang Y, Lv J, Jiang S, Ma Z, Wang D, Hu W, et al. The emerging role of toll-like receptor 4 in myocardial inflammation. Cell Death Dis. 2016;7:e2234.

25. Nishida K, Otsu K. Inflammation and metabolic cardiomyopathy. Cardiovasc Res. 2017;113:389.

26. Abel ED, Litwin SE, Sweeney G. Cardiac remodeling in obesity. Physiol Rev. 2008;88:389-419.

27. Haiyan X, Barnes GT, Qing Y, Guo T, Daseng Y, Chou CJ, et al. Chronic inflammation in fat plays a crucial role in the development of obesity-related insulin resistance. J Clin Investig. 2003;112:1821-30.

28. Vasan RS. Cardiac function and obesity. Heart. 2003;89:1127-9.

29. Sletten AC, Peterson LR, Schaffer JE. Manifestations and mechanisms of myocardial lipotoxicity in obesity. J Intern Med. 2018;284:478-91.

30. Qian $Y$, Zhang Y, Zhong $P$, Peng $K, X u$ Z, Chen $X$, et al. Inhibition of inflammation and oxidative stress by an imidazopyridine derivative $x 22$ prevents heart injury from obesity. J Cell Mol Med. 2016;20:1427-42.

31. Ge CX, Xu MX, Qin YT, Gu TT, Lou DS, Li Q, et al. Endoplasmic reticulum stressinduced irhom2 up-regulation promotes macrophage-regulated cardiac inflammation and lipid deposition in high fat diet (hfd)-challenged mice: Intervention of fisetin and metformin. Free Radic Biol Med. 2019;141:67-83.

32. Cao J, Singh SP, McClung JA, Joseph G, Vanella L, Barbagallo I, et al. Eet intervention on wnt1, nov, and ho-1 signaling prevents obesity-induced cardiomyopathy in obese mice. Am J Physiol Heart Circ Physiol. 2017;313:H368-h80.

33. Singh MV, Cicha MZ, Meyerholz DK, Chapleau MW, Abboud FM. Dual activation of trif and myd88 adaptor proteins by angiotensin ii evokes opposing effects on pressure, cardiac hypertrophy, and inflammatory gene expression. Hypertension. 2015;66:647-56.

34. Przemyslaw B, Gabriela K, Thomas D, Marty RR, Alan V, Corinne B, et al. Myeloid differentiation factor-88/interleukin-1 signaling controls cardiac fibrosis and heart failure progression in inflammatory dilated cardiomyopathy. Circ Res. 2009;105:912-20. 